\title{
Interference Phenomena and Whispering-Gallery Modes of Synchrotron Radiation in a Cylindrical Wave-Guide
}

\author{
Sándor Varró \\ Wigner Research Centre for Physics \\ of the Hungarian Academy of Sciences \\ Hungary
}

\section{Introduction}

In 'usual lasers' the light amplification is a consequence of stimulated emission stemming from bound-bound quantum transitions in the active medium (e.g. gas or vapour atoms, molecules, or radiation centers in a solid), on the other hand, in free electron lasers (FELs) the radiation is produced in free-free transitions, taking place in static magnetic fields. In a homogeneous static magnetic field an electron (or other charged particle) moves along an ideal circular (or, in general, helical) trajectory, if the radiation damping is negligible, and emits a 'broad-band radiation' containing all the higher harmonics of its frequency of revolution. The characteristics of this ideally periodic radiation field has been first analysed by Schott [1]. Later several refinements and asymtotic formulae have been derived for describing the quasi-continuous radiation (which in the meantime received the name 'synchrotron radiation') of ultrarelativistic electrons moving along an instantaneous circular orbit (see e.g. Schwinger [2]). A very detailed analysis of this radiation can be found in the book by Sokolov and Ternov [3], which deals with this phenomenon both in the frame of classical electrodynamics and in the frame of relativistic quantum mechanics. By the beginning of the eighties of the last century the synchrotron radiation has become a widely used experimental tool in many branches of science and technology [4].

The idea of using periodic magnetic fields (undulators, wigglers) for properly bending the trajectories of the (ultra)relativistic electrons, and generating coherent radiation is due to Motz [5]. Besides working out the classical theory, he, with his coworkers, made the first experimental demonstrations, too. In these experiments a $100-\mathrm{MeV}$ electron beam from the Stanford linear accelerator passed through the undulator, and besides millimeter waves, light radiated by the beam was also observed [6]. Later Schneider [7] found, by using a phenomenological rate equation approach, that the absorption of radiation around the cyclotron resonance of an electron may go over to negative absorption (stimulated emission), if we take into account the relativistic kinematics of the electrons. His analysis was based on the transitions between the Landau levels of electrons in a homogeneous magnetic field. In 1971 Madey's work [8] gave a new impetus to the research on generation of coherent radiation by free electron beams. He used the Weizsacker-Williams approximation (or in other words, the method of equivalent photons, see e.g. Heitler [9]) to 
calculate the gain due to the induced emission of radiation into a single electromagnetic mode parallel to the motion of a relativistic electron through a periodic transverse static magnetic field. He has proved that "Finite gain is available from the far-infrared through the visible region raising the possibility of continuously tunable amplifiers and oscillators at these frequencies with the further possibility of partially coherent radiation sources in the ultraviolet and $x$-ray regions to beyond $10 \mathrm{keV."[8].} \mathrm{In} \mathrm{this} \mathrm{description} \mathrm{the} \mathrm{periodic}$ magnetic field is represented by an 'equivalent photon beam' in the nearly comoving frame of the relativistic electrons. By going over to this frame, the macroscopic period (on the order of centimeters) of the magnet is down-converted, due to the Lorentz contraction, to microns or even shorter wavelengths, depending on the velocity of the electron. According to the appropriate Lorentz transformation, this periodic field is essentially equivalent to a zero-mass-shell true radiation field, which is back-scattered on the electron. In this frame the amplification may come from the stimulated Compton scattering, if the radiation is coupled back by mirrors, e.g. in a Fabry-Perot arrangement. Concerning the further development of the theory, it was a remarkable result in Madey's work [8] that, though the quantum kinematics of the Compton process has been used in the analysis, the final gain formula does not contain Planck's constant in the relevant regime of the parameters. In fact, most part of the later analyses of FELs were relying on classical electrodynamics and statistics (see e.g. [10-12]). Really, the first true laser operations [13] in the infrared, at $\lambda \sim 3.4 \mu \mathrm{m}$ and in the visible [14] at wavelength $\lambda \sim 0.65 \mu \mathrm{m}$ could be satisfactorly described in the framework of the cassical theory. From the conceptual point of view, this has been in good accord with an earlier analysis of Borenstein and Lamb on the "classical laser"[15]. Nevertheless, Madey [8] has already made some estimates concerning the photon statistics of the proposed FEL. Also in a later special issue on free electron lasers Chen and Madey [16] concluded that "Both the experiment data and the simulations indicate that the reduction of phase noise are directly associated with electron bunching. In fact, higher degree of electron bunching leads to less phase fluctuations in the optical field. The conclusion is that our study has once again supported the idea of imparting statistical properties from radiating particles to radiated photons. Hence the generation of phase squeezed light out of the FEL radiation is anticipated, given the reduction of electron phase fluctuations due to the FEL bunching mechanism." A more general analysis of related questions has been given by Tanabe [17] in this same special issue. According to his conclusions "An ideal single-mode amplitudestabilized gas laser operating well above its threshold could produce coherent state, however, many so-called 'coherent' light may possess only the first-order coherence. Some experimental efforts have been made for spontaneous emission; however, no experimental investigation was made for FEL stimulated emission either in exponential gain regime or in saturated one." In fact, there have already been theoretical results published, according to which the interactions of free electrons with quantized radiation fields lead to non-classical states [18-21], like squeezed coherent states or number-phase minimum-uncertainty states. Both in theory and in practice, for the characterization of the statistical properties of such radiations, the higher-order correlation functions have to be studied, as is done in Hanbury Brown and Twiss type experiments [22-23]. Recent measurements [24-25] on undulator radiation in the x-ray regime have shown that the spontaneous signal produces positive second order (intensity-intensity) correlation, similar to that of thermal radiation. This is set to be the consequence of the chaotic spatial distributions of the electrons. For the FEL a sort of 'phase-squeezed state' has been 
expected by Chen and Madey [16] earlier, on the other hand, extremely short (attosecond) pulses produced in very high-order harmonic generation processes of atoms or solids [2627], can certainly be reasonably represented by quantum mechanical phase eigenstates [28]. At present it seems that the higher-order quantum correlation properties of the existing FEL sources [29-31] do not play a significant role in the applications.

We have seen above that he cyclotron and synchrotron radiation in free space has long been thoroughly studied both theoretically and experimentally. The feedback of the radiation, in case of a true laser system is usually secured by a Fabry-Perot type linear resonator arrangement. Besides, already in the sixties of the last century, in the context of cyclotron masers [32] and cavity electrodynamics, more general cavity effects have also been studied [33-34]. In the cyclotron maser the fundamental or low harmonics are generated, on the other hand, in the synchrotron radiation very high harmonics are dominant. The question naturally emerges whether could one somehow feed back these high harmonics in order to have stimulated emission and manage perhaps lasing also. This idea dates back to the principle of operation of the so-called 'halo laser' [35], on the basis of which twodimensional stimulated emission and planar laser action in the whole $2 \pi$ angle have been achieved. In this device the laser active dye material in a cylindrical cuvette is pumped from below, and the feedback is secured by partial radial and oblique reflections on the wall of the cuvette. Besides, the whispering-gallery modes of light may have also served for energy storage. The functioning of this system has clearly illustrated that the linear cavity and very small divergence is not a necessary condition to the laser action. We have studied [36-37] a slightly analogous feedback mechanism, where the radiation, emanating tangentially from an electron, is coupled back by a cylindrical mirror. The radiation follows the electron in the form of very high angular momentum whispering gallery modes. The present chapter is devoted to the discussion of the case in which the trajectories of the gyrating ultrarelativistic electrons are completely surrounded by a coaxial mirror, in a wave-guide configuration. We will show that, if the ratio of the the cylinder's radius and radius of the electron's trajectory satisfies certain geometrical resonance conditions, then the resonance practically for all very high harmonics can be achieved. As a general remark, we would like to note that recently there has been a wide-spreading interest in the physics of electromagnetic whispering-gallery modes in nanostructures [38-39]. For instance, in ref [38] it has been demonstrated that e.g. crystalline optical whispering-gallery resonators with very high $Q$ factors can be constructed, and used for studying nonlinear processes, owing to the large field concentration.

In Section 2 the combined initial-value and boundary-value problem of the relevant Maxwell equations are solved exactly on the basis of the suitable Green's function, and the modal structure of the synchrotron radiation in the cylindrical mirror is analysed. The direct radiation reaction will be left out of consideration, however the effect of damping at resonance will be briefly discussed. In Section 3 we prove the existence of a 'broad-band resonance' where an accumulation process is taking place, due to which the intensity of the resulting radiation can be considerably increased. The present analysis aims to show that, if the electron meets with its own radiation field emitted earlier in a tangential narrow cone, and reflected back by the cylindrical mirror, then a constructive interference shows up between this retarded self-field and the actually emitted radiation field. This arrangement could perhaps serve as a basis for constructing a compact "disk-synchrotron-lasers" radiating in a plane. In section 4 we shall briefly summarize our results. 


\section{Excitation of cylindrical wave-guide modes by an ultrarelativistic electron}

In the present section we give an exact solution of Maxwell's equations driven by an ultrarelativistic electron gyrating inside of a perfectly reflecting cylindrical wave-guide.

The transverse position of the electron moving in the homogeneous magnetic field $\vec{e}_{z} B_{0}$ has the Cartesian components

$$
x(t)=r_{0} \cos \left(\omega_{0} t+\varphi_{0}\right), y(t)=r_{0} \sin \left(\omega_{0} t+\varphi_{0}\right), z(t)=0,
$$

where $r_{0}=v / \omega_{0}, \omega_{0}=\omega_{c} / \gamma$ with $\omega_{c}=|e| B_{0} / m c$ being the cyclotron frequency, $v$ denotes electron's velocity and $\gamma \equiv\left(1-\beta^{2}\right)^{-1 / 2}, \beta \equiv v / c$. We assume that the longitudinal component of the electron's velocity is zero, i.e. $v_{z}=0$, and the gyration takes place in the $z=0$ plane. The charge density $\rho$ and the current density $\vec{j}$, associated to the trajectory of one single electron with initial phase $\phi_{0}$ given by Eq. (1), can be conveniently expressed in cylindrical coordinates

$$
\{\rho(r, \varphi, z ; t), \vec{j}(r, \varphi, z ; t)\}=\left\{1, \overrightarrow{v e}_{\varphi}\right\} e r^{-1} \delta\left(r-r_{0}\right) \delta\left[\varphi-\left(\omega_{0} t+\varphi_{0}\right)\right] \delta(z) u(t)
$$

where we have also introduced the unit step function $u(t)$, describing a sharp switching-on of the interaction. Equations (1) and (2) correspond to a highly idealistic situation for many reasons. Because in reality no perfectly homogeneous and stationary magnetic fields can be sustained over large spatio-temporal regions, exactly planar and circular trajectories of charges can never be secured, and, moreover the position of the guiding center cannot be fixed up to an arbitrary accuracy. The production and injection of a perfectly monoenergetic electron beam is impossible, either. The radiation loss necessarily distorts the trajectory, and the reacceleration cannot be solved without causing additional oscillations. In short, the spatio-temporal inhomogenities and spectral imperfections of both the boundary conditions and of the charged particle beams, of course, do not allow to prepare and sustain such an ideal current distribution shown in Eq. (2). The 'fragility' of this external current density may be a subject of a separate study, like the investigation of the possible bunching effect caused by the feedback of the radiation which would result in superradiance and lasing. In the present we shall not consider these important questions, rather we concentrate on the simplest, but exactly solvable part of the radiation problem.

In order to obtain a physically meaningful solutions of Maxwell's equations driven by the densities given by Eq. (2) inside the cylinder, we have to take into account the boundary conditions $[\vec{n} \times \vec{E}]_{C}=0$ and $[\vec{n} \cdot \vec{B}]_{C}=0$, where the subscript $C$ symbolizes the boundary of the cylinder of radius $a$ (larger than $r_{0}$ ), and $\vec{n}$ is the outward unit normal of it. In words this means that the tangential component of the electric field strength $\vec{E}$ and normal component of the magnetic induction $\vec{B}$ must vanish at the surface of the cylinder (at any vertical position $z$ ). Thus $\vec{E}$ and $\vec{B}$ are expanded into a superposition of the so-called crosssectional vector eigenfunctions [40-41],

$$
\vec{E}=\sum_{m p} a_{m p} \vec{\nabla}_{\perp} \Phi_{m p}+\sum_{n s} b_{n s} \vec{e}_{z} \times \vec{\nabla}_{\perp} \Psi_{n s}+\vec{e}_{z} \sum_{m p} c_{m p} \Phi_{m p}
$$




$$
\vec{B}=\sum_{m p} \alpha_{m p} \vec{e}_{z} \times \vec{\nabla}_{\perp} \Phi_{m p}+\sum_{n s} \beta_{n s} \vec{\nabla}_{\perp} \Psi_{n s}+\vec{e}_{z} \sum_{n s} \gamma_{n s} \Psi_{n s}
$$

The symbol $\vec{\nabla}_{\perp}=(\partial / \partial x, \partial / \partial y, 0)$ in Eqs. (3a-b) denotes the transverse nabla vector. In Eqs. $(3 \mathrm{a}, \mathrm{b}) \Phi_{m p}$ and $\Psi_{n s}$ are Dirichlet and Neumann eigenfunctions satisfying the scalar Helmholtz equation $\left(\nabla_{\perp}^{2}+k^{2}\right) f=0$ with eigenvalues $k_{m p}$ and $k_{n s}$, respectively. The unknown coefficients $a_{m p}, b_{n s}, c_{m p}$ and $\alpha_{m p}, \beta_{n s}, \gamma_{n s}$ are to be determined as functions of time $(t)$ and vertical position $(z)$. According to the boundary condition $\left[\Phi_{m p}\right]_{C}=0, \Phi_{m p}$ must have the form

$$
\Phi_{m p}=J_{m}\left(x_{m p}(r / a)\right)\left\{\begin{array}{c}
\sin (m \varphi) \\
\cos (m \varphi)
\end{array}\right\}, J_{m}\left(x_{m p}\right)=0
$$

where $x_{m p}$ is the $p$-th root of the ordinary Bessel function of first kind $J_{m}$ of order $m$. Because of the Neumann boundary condition, $\left[\partial \Psi_{n s} / \partial r\right]_{C}=0$, we have

$$
\Psi_{n s}=J_{n}\left(y_{n s}(r / a)\right)\left\{\begin{array}{c}
\sin (n \varphi) \\
\cos (n \varphi)
\end{array}\right\}, J_{n}^{\prime}\left(y_{n s}\right)=0
$$

where $y_{n s}$ is the $s$-th root of the derivative $J_{n}^{\prime}$ of the the ordinary Bessel function of first kind of order $n$. The eigenvalues of the corresponding wave numbers are $k_{m p}=x_{m p} / a$ and $k_{n s}=y_{n s} / a$, respectively, where $a$ is radius of the cylinder. By taking into account the orthogonality property of the cross-sectional eigenfunctions, we can derive from the inhomogeneous Maxwell equations two sets of coupled first order differential equations for the expansion coefficients. The set $\left\{a_{m p}, \alpha_{m p}, c_{m p}\right\}$ is responsible for the dynamics of the TM and longitudinal components of the electromagnetic field. On the other hand, the dynamics of the TE components is governed by the set $\left\{b_{n s}, \beta_{n s}, \gamma_{n s}\right\}$. It can be shown that $\alpha_{m p}(z=0, t)=0$ and $c_{m p}(z=0, t)=0$, moreover, in the case we shall discuss below $a_{m p}$ vanishes to a good approximation at any vertical position. This means that in the plane of the electron gyration only the TE modes are excited, and that is why henceforth we shall be dealing only with the behaviour of the TE modes.

The coupled system of equations for $b_{n s}, \beta_{\mathrm{ns}}$ and $\gamma_{\mathrm{ns}}$ reads

$$
\begin{gathered}
\frac{\partial \beta_{n s}}{\partial z}-\frac{1}{c} \frac{\partial b_{n s}}{\partial t}-\gamma_{n s}=\frac{4 \pi}{c} \frac{1}{N_{n s}^{2}} \int d^{2} s \vec{j} \cdot\left(\vec{e}_{z} \times \vec{\nabla}_{\perp} \Psi_{n s}\right) \\
\frac{\partial b_{n s}}{\partial z}-\frac{1}{c} \frac{\partial \beta_{n s}}{\partial t}=0 \\
b_{n s}-\frac{1}{c k_{n s}^{2}} \frac{\partial \gamma_{n s}}{\partial t}=0
\end{gathered}
$$

where $N_{n s}^{2}=\left(\pi / \varepsilon_{n}\right) J_{n}^{2}\left(y_{n s}\right)\left(y_{n s}^{2}-n^{2}\right)$, and $\varepsilon_{1}=1$ and $\varepsilon_{n}=2$ for $n=2,3, \ldots$. The integration on the rhs of Eq. (5a) is to be evaluated over the cross-section of the cylinder. Having eliminated the functions $\beta_{n s}$ and $\gamma_{n s}$ from Eqs. $(5 \mathrm{a}, \mathrm{b}, \mathrm{c})$ we arrive at an inhomogeneous Klein-Gordon equation for $b_{n s}(z, t)$, 


$$
\left(\frac{\partial^{2}}{\partial z^{2}}-\frac{1}{c^{2}} \frac{\partial^{2}}{\partial t^{2}}-k_{n s}^{2}\right) b_{n s}=B_{n s} \delta(z) f_{n}^{\prime}(t) / c
$$

where

$$
B_{n s} \equiv 4 e \beta \frac{\left(y_{n s} / a\right) J_{n}^{\prime}\left(y_{n s} r_{0} / a\right) \varepsilon_{n}}{J_{n}^{2}\left(y_{n s}\right)\left(y_{n s}^{2}-n^{2}\right)}, f_{n}(t) \equiv\left\{\begin{array}{l}
\sin \left[n\left(\omega_{0} t+\varphi_{0}\right)\right] \\
\cos \left[n\left(\omega_{0} t+\varphi_{0}\right)\right]
\end{array}\right\} u(t)
$$

The Green's function of Eq. (6a) can be derived by using standard methods

$$
g(t, z)=-\frac{c}{2} J_{0}\left(c k_{n s} \sqrt{t^{2}-z^{2} / c^{2}}\right) u\left(t-\frac{|z|}{c}\right), t \equiv t_{1}-t_{2}, z \equiv z_{1}-z_{2}
$$

With the help of the Green's function in Eq. (7), the solution of Eq. (6a) can be determined by straighforward integrations. The general explicit form of $b_{n s}$ is a complicated expression, however for large values of $t$ a relatively simple expression can be derived (for short, we present only the upper component stemming from the sine oscillations):

$$
\begin{aligned}
& -2 b_{n s}(z, t) / B_{n s}=J_{0}\left(\omega_{n s} \sqrt{t^{2}-z^{2} / c^{2}}\right)\left(\sin \varphi_{0}\right) u\left(t-\frac{1}{c}|z|\right) \\
& +\frac{v_{n s} u\left(v_{n s}-1\right)}{\sqrt{v_{n s}^{2}-1}} \sin n\left[\omega_{0}\left(t-\frac{1}{c} \sqrt{v_{n s}^{2}-1}|z|\right)+\varphi_{0}\right] \\
& +\frac{v_{n s} u\left(1-v_{n s}\right)}{\sqrt{1-v_{n s}^{2}}}\left(\cos n\left(\omega_{0} t+\varphi_{0}\right)\right) \exp \left[-\frac{n \omega_{0}}{c} \sqrt{1-v_{n s}^{2}}|z|\right]
\end{aligned}
$$

where $v_{n s} \equiv n \omega_{0} / \omega_{n s}$ with $\omega_{n s} \equiv c k_{n s}$ being the TE eigenfrequencies. The first term on the rhs of Eq. (8) represents a (transient) precursor with front velocity $c$, which vanishes as $1 / \sqrt{t}$. The second and the third terms correspond to above-cutoff and below-cutoff waves, respectively, the latter being bound to the $z=0$ plane. At the exact resonances $v_{n s}=1$, Eq. (8) loses its validity, and $b_{n s}$ has a qualitatively different form. For short, we present only the upper component of $b_{n s}$ taken at the $z=0$ plane (of the electron's trajectory):

$$
-2 b_{n s} / B_{n s}=J_{0}\left(n \omega_{0} t\right)\left(\sin n \varphi_{0}\right)+\left(n \omega_{0} t\right)\left[J_{0}\left(n \omega_{0} t\right)\left(\cos n \varphi_{0}\right)-J_{1}\left(n \omega_{0} t\right)\left(\sin n \varphi_{0}\right)\right]
$$

On the basis of the asymptotic behaviour of the Bessel functions, it can be shown that for large $t$ times $b_{n s}$ diverge as $\sqrt{t} \times$ (oscillatory function). Of course, since some sort of damping is always present in physical systems, such a divergence is not realistic. If we introduce the phenomenological damping term $\left(-k_{n s} / c Q_{n s}\right)\left(\partial b_{n s} / \partial t\right)$ into the Klein-Gordon equation on the lhs of Eq. (6), then the solution has a similar structure as that shown in Eq. (8), but with the essential difference that the potential unphysical divergences are replaced by finite resonance terms. The oscillatory parts will contain resonance denominators of the form $\left[\left(v_{n s}^{2}-1\right)^{2}+v_{n s}^{2} / Q_{n s}^{2}\right]^{1 / 4}$, where $Q_{n s}$ is spectral Q-factor related to the assumed finite conductivity of the cylinder wall. Thus, close to resonance the amplitudes are increased by a factor of $\sqrt{Q_{n s}}$. For the high harmonics, we are interested in, $Q_{n s}$ can be well approximated 
by $a / \delta_{n s}$, where $\delta_{n s}=\left(2 / \mu \sigma \omega_{n s}\right)^{1 / 2}$ is the skin depth for a spectral component. For example, for silver $\delta_{\omega} \approx 6 \times 10^{-6} \mathrm{~cm}$ for $\omega / 2 \pi \approx 10^{10} \mathrm{~Hz}$. In the optical region $\delta_{\omega}$ can well be of two orders of magnitude smaller, thus $Q_{\omega}$ can be very large if $a$ is of order of meters, say.

\section{Resonance conditions}

In the present section we study the question of under what conditions simultaneous resonance can be reached for the 'most of the higher harmonics' in the synchrotron radiation in the cylindrical mirror. The geometrical arrangement we are interested in is shown in Fig. 1. On geometrical resonance condition we mean that the radii of the electon's trajectory and of the cylinder are adjusted in such a way, that a signal emanating tangentially at point $A$, after reflection, gets back to the electron's trajectory at point $B$ exactly at that time when the electron (possibly after $N \geq 1$ complete revolutions, or even 'almost immediately') gets to the same point $B$. It is clear that, if once this condition is satisfied for the pair of points $A$ and $B$, then it will be satisfied for the pair $A^{\prime}$ and $B^{\prime}$, which can be obtained by rotating the pair $A$ and $B$ by an arbitrary angle. In this way the electron 'after a while' will continuously move 'in phase' in its own retarded radiation field which has been emitted earlier at different points on the trajectory. We think that this arrangement would secure an effective feedback for obtaining stimulated emission. Needless to say, the correctness of the expectation suggested by this intuitive picture (which was originally just the starting point of the present investigation) should be checked on the basis of the accurate analytic treatment. By simple kinematic considerations it can be shown that, if the geometrical resonance condition holds, then the ratio $a / r_{0}$ satisfies the following transcendental equation

$$
\beta \sqrt{x^{2}-1}-\arccos (1 / x)=N \pi, x \equiv a / r_{0}
$$

where $N$ is the number of complete revolutions of the electron before the first ecounter with its own radiation field after one reflection. For $N=1$ we obtain approximately $a / r_{0} \cong 3 \pi / 2$, and for $N=0$ we have $a / r_{0} \cong 1+3 / 4 \gamma^{2}$. It is clear that for an ultrarelativistic electron the latter 'zeroth resonant condition' can be satisfied if $a$ is only slightly larger than the radius of the trajectory, i.e. the electron moves very close to the inner surface of the cylinder. If we assume $r_{0} \approx 10 \mathrm{~cm}$ in a strong confining magnetic field, then the the first resonance condition can be satisfied in a cylinder of radius $a \approx 47 \mathrm{~cm}$. Henceforth we shall study the case $N=1$.

The wave resonance condition $1=v_{n s} \equiv n \omega_{0} / \omega_{n s}=\left(n / y_{n s}\right)\left(\beta a / r_{0}\right)$, on the basis of the previous section, can be studied by using the asymptotic form of the zeros $y_{n s}$ of the derivatives $J_{n}^{\prime}$ of the Bessel functions $J_{n}$. Here we restrict the discussion to the case when not only $n$ but also the $s$ are large. (It can be shown that if $s$ considerably differs from $n$, then the resonance cannot be reached.) For large $n$ we have

$$
\frac{a}{r_{0}} \cong \beta \frac{a}{r_{0}}=\frac{y_{n s}}{n}=z(\zeta)+O\left(\frac{1}{n^{2}}\right), \sqrt{z^{2}-1}-\arccos (1 / x)=\frac{2}{3}(-\zeta)^{3 / 2}, \zeta=n^{-3 / 2} a_{s}^{\prime} .
$$



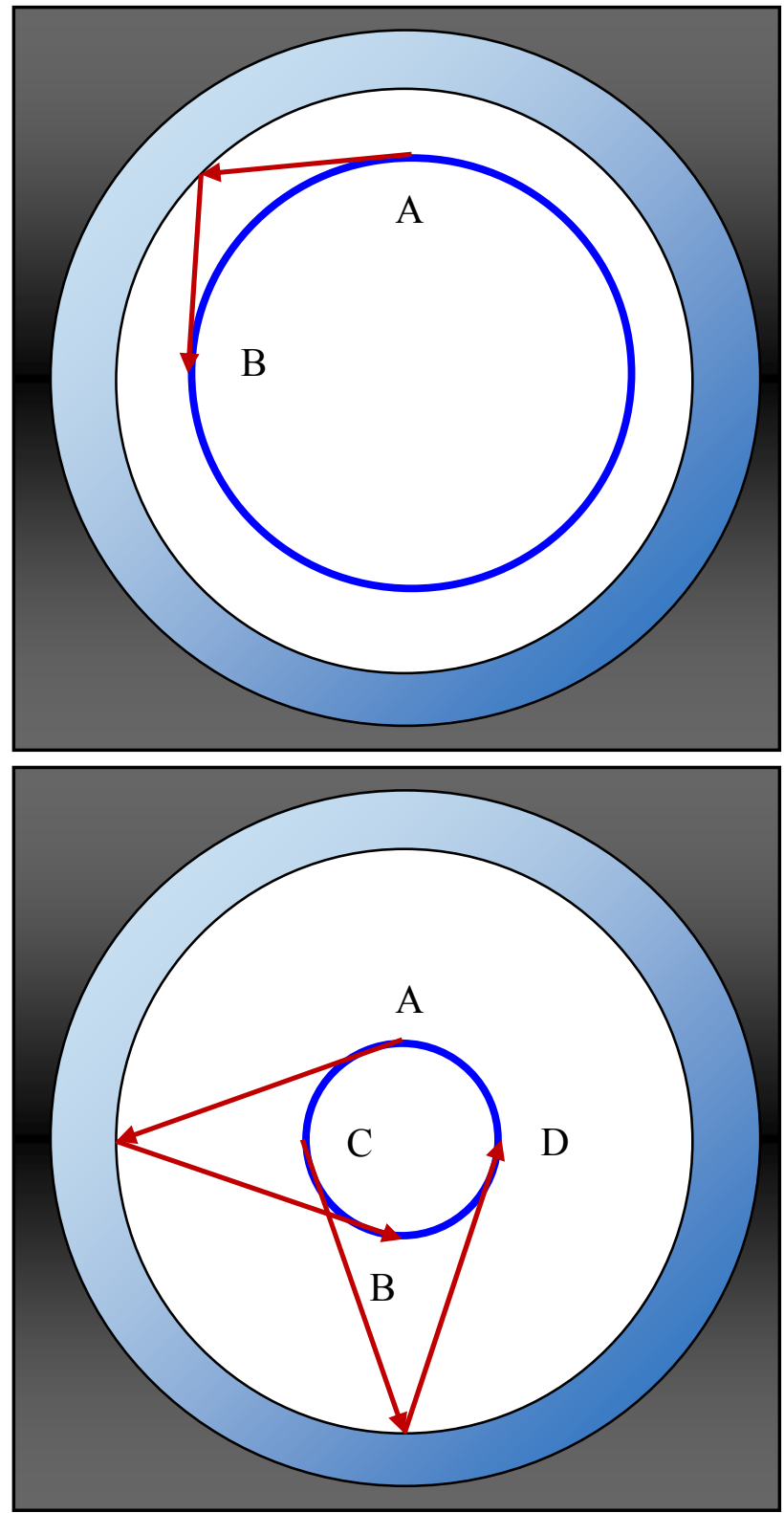

Fig. 1. A ray of radiation emanating tangentially within a narrow cone from the electron at point $A$, gets reflected on the cylindrical mirror and arrives at point $B$ exacly at the time when the electron arrives there. Left: illustrates the zeroth geometrical resonance condition $(\mathrm{N}=0)$. Right: illustrates the first resonance condition $(\mathrm{N}=1)$. In the latter case, after one complete revolution the electron encounters with its own radiation field (which was emitted tangentially one turn earlier). For further explanation see the main text. 
The function $z(\zeta)$ is the inverse function defined by the second equation of Eq. (11), with $a_{s}^{\prime}$ being the $s$-th negative zeros of the Airy function. Since $n$ is very large, $z=x$, and because $\beta=v / c$ is practically unity, the left hand sides of Eq. (10) coincides with the left hand side of the second equation of Eq. (11). Now, for large $s$ values the zeros can be approximated by the analytic formula $-\zeta=(3 \pi s / 2 n)^{2 / 3}$. By having taken this relation into account, we can easily check that, if the geometrical resonance condition is secured, then the wave resonance condition in Eq. (10) becomes an identity for $s=n$. As a consequence, the wave resonance condition is independent of the $n$-values, provided these are large enough. This means that (if $Q_{n s}$ is a smooth function of $n$ ) there exist a broad band of the spectrum which is uniformly 'lifted-up' due to these resonances. This resonant accumulation process can be interpreted as a result of the constructive interference between the radiation actually emitted and the self-radiation emitted earlier and fed back (by reflection) to the actual position of the electron.

\section{Summary}

We have discussed the characteristics of the synchrotron radiation emitted by ultrarelativistic electrons in the interior of a coaxial cylindrical mirror. This is an unconventional geometrical arrangement, where the "superradiance " may be realized in two dimensions. In the first section we have summarized the early history and basic concepts of free-electron lasers, and outlined briefly some still partly open questions concerning the coherence properties of such sources of radiation. In the main part of the present chapter, in sections 2 and 3 we have analysed the characteristics of the synchrotron radiation emitted by a single ultrarelativistic electron in the interior of a coaxial cylindrical mirror. The combined initial-value and boundary-value problem of the Maxwell equations have been solved exactly, but neither the radiation reaction, nor the role of spatio-temporal imperfections have been discussed. It was shown that near the plane of the electron's gyration mostly the TE modes are excited, and the effect of damping at resonance has also been briefly discussed. In section 3 we have introduced the concept of geometrical resonance, whose notion is based on a simple ray construction. It was shown that if this condition is satisfied, then the exact wave resonance condition does not depend on the excitation indeces of the very high harmonics. At such a 'broad-band resonance' there is an accumulation process taking place, due to which the intensity of the resulting radiation can be increased by orders of magnitudes.

The present analysis supports our original physical picture according to which, if, in the discussed geometrical arrangement, the electron meets with its own radiation field emitted earlier in a tangential narrow cone, and reflected back by the mirror, then there is a constructive interference between this retarded self-field and the actually emitted radiation field.

\section{Acknowledgement}

This work has been supported by the Hungarian National Scientific Research Foundation OTKA, Grant No. K73728, and by the National Development Agency, Grant No. ELI_09-12010-0010, Helios Project. 


\section{References}

[1] G. A. Schott, Electromagnetic Radiation (Cambridge University Press, Cambridge, 1912)

[2] J. Schwinger, On the classical radiation of accelerated electrons. Phys. Rev. 75, 1912-1925 (1949)

[3] A. A. Sokolov and I. M. Ternov, Synchrotron Radiation (Akademie-Verlag GmbH, Berlin, 1968)

[4] H. Winnick and S. Doniach, Synchrotron Radiation Reseach (Plenum Press, New York, 1980)

[5] H. Motz, Application of the radiation from fast electron beams. J. Appl. Phys. 22, 527-535 (1951). See also H. Motz, Errata. J. Appl. Phys. 22, p. 1217 (1951)

[6] H. Motz, W. Thon and R. N. Whitehurst, Experiments on radiation by fast electron beams. J. Appl. Phys. 24, 826-833 (1953).

[7] J. Schneider, Stimulated emission of radiation by relativistic electrons in a magnetic field. Phys. Rev. Lett. 2, 504-505 (1969)

[8] J. M. J. Madey, Stimulated emission of Bremsstrahlung in a periodic magnetic field. J. Appl. Phys. 42, 1906-1913 (1971).

[9] W. Heitler, The Quantum Theory of Radiation (Clarendon Press, Oxford, England, 1960), p. 414.

[10] A. Bambini, A. Renieri and S. Stenholm, Classical theory of the free-electron laser in a moving frame. Phys. Rev. A 19, 2013-2025 (1979)

[11] F. A. Hopf, T. G. Kuper, G. T. Moore and M. O. Scully, in Free-electron generation of coherent radiation. Eds. S. F. Jacobs, M. S. Piloff, M. Surgent III, M. O. Scully and R. Spitzer (Addison-Wesley, London, 1980) pp. 31-57.

[12] W. B. Colson and S. K. Ride, in Free-electron generation of coherent radiation. Eds. S. F. Jacobs, M. S. Pieloff, M. Surgent III, M. O. Scully and R. Spitzer (Addison-Wesley, London, 1980) pp. 377-412.

[13] D. A. G. Deacon, L. R. Elias, J. M. J. Madey, G. J. Ramian, H. A. Schwettmann and T. I. Smith, First operation of a free electron laser. Phys. Rev. Lett. 38, 892-894 (1977)

[14] M. Billardon, P. Elleaume, J. M. Ortega, C. Bazin, M. Bergher, M. Velghe, Y. Petroff, D. A. G. Deacon, K. A. Robinson and J. M. J. Madey, First operation of a storage-ring free electron laser. Phys. Rev. Lett. 51, 1652-1655 (1983)

[15] M. Borenstein and W. E. Lamb, Jr., Classical laser. Phys. Rev. A 5, 1298-1311 (1972)

[16] T. Chen and J. M. J. Madey, Study of squeezed state on free electron lasers, In J. Xie and $X$. Du (Guest Editors), Proceedings of the The Nineteenth International Free Electron Laser Conference and the Fourth FEL Users' Workshop, 18-21 August 1997 in Beijing, China. Nucl. Inst. Meth. Phys. Res. A-407, 203-209 (1998)

[17] T. Tanabe, Photon statistics of various radiation sources. Nucl. Inst. Meth. Phys. Res. A407, 252-256 (1998)

[18] J. Bergou and S. Varró, Nonlinear scattering processes in the presence of a quantised radiation field: II. Relativistic treatment. J. Phys. A: Math. Gen. 14, 1469-1482 (1981)

[19] W. Becker, K. Wódkiewicz and M. S. Zubairy, Squeezing of the cavity vacuum by a charged particle. Phys. Rev. A 36, 2167-2170 (1987)

[20] S. Varró, Entangled photon-electron states and the number-phase minimum uncertainty states of the photon field. New J. Phys. 10, 053028 (2008) 
[21] S. Varró, Entangled states and entropy remnants of a photon-electron system. Phys. Scr. T140, 014038 (2010).

[22] S. Varró , Correlations in single-photon experiments. Fortschritte der Physik-Prog. Phys. $56,91-102$ (2008)

[23] S. Varró , The role of self-coherence in correlations of bosons and fermions in counting experiments. Notes on the wave-particle duality. Fortschritte der Physik-Progress of Physics 59, 296-324 (2011)

[24] M. Yabashi, K. Tamasaku and T. Ishikawa, Intensity interferometry for the study of $\mathrm{x}-$ ray coherence. Phys. Rev. A 69, 023813 (2004)

[25] E. Ikonen, M. Yabashi and T. Ishikawa, Excess coincidences of reflected and refracted $\mathrm{x}$ ray from a synchrotron-radiation beamline. Phys. Rev. A 74, 013816 (2006)

[26] Krausz, F. \& Ivanov, M. (2009). Attosecond physics. Rev. Mod. Phys. 81, 163-234.

[27] S. Varró, Intensity effects and absolute phase effects in nonlinear laser-matter interactions. In F. J. Duarte (Ed.), Laser Pulse Phenomena and Applications (InTech, Rijeka, 2010) pp. 243-266.

[28] S. Varró, Attosecond shot noise. 41th Winter Colloquium on the Physics of Quantum Electronics (PQE-41, 2-6 January 2011, Snowbird, Utah, USA) p. 132.

[29] S. Kahn, Free-electron lasers. (a tutorial review) Journal of Modern Optics 55, 3469-3512 (2008)

[30] H. Chapman, J. Ullrich and J. M. Rost, Intense x-ray science: the first 5 years of FLASH. (Editorial of the special issue FLASH reseach) J. Phys. B: At. Mol. Opt. Phys. 43 (2010) 190201 (1pp)

[31] J. R. Schneider, FLASH - from accelerator test facility to the first single-pass soft x-ray free-electron laser. J. Phys. B: At. Mol. Opt. Phys. 43 (2010) 194001 (9pp)

[32] J. L. Hirschfield and J. M. Wachel, Electron cyclotron maser. Phys. Rev. Lett. 12, 533-536 (1964)

[33] L. S. Brown, K. Helmerson and J. Tan, Cyclotron motion in a spherical microwave cavity. Phys. Rev. A 34, 2638-2645 (1986)

[34] S. J. Han, Stability of a rotating relativistic electron beam in a waveguide near the cyclotron resonance. Phys. Rev. A 35, 3952-3955 (1987)

[35] Z. Gy. Horváth and S. Varró, Modes in wall-reflection planar halo lasers. Optica Acta 32, 1125-1144 (1985)

[36] S. Varró, Synchrotron radiation in the presence of a perfect cylindrical mirror. Proceedings of the Third European Particle Accelerator Conference (Berlin, 24-28 March, 1992) (Editors: H. Henke, H. Homeyer and Ch. Petit-Jean-Genaz, Edition Frontieres, 1992) Volume 1, pp 209-211.

[37] S. Varró, Synchrotron radiation in the presence of a perfect cylindrical mirror. Note on the interference of the direct radiation and the high-order whispering-gallery modes. arXiv:1101.5811 (physics.acc-ph); (math-ph) (2011)

[38] V. S. Ilchenko, A. A. Savchenko, A. B. Matsko and L. Maleki, Nonlinear optics and crystalline whispering gallery mode cavities. Phys. Rev. Lett. 92, 043903 (2004)

[39] J. U. Fürst, D. V. Strekalov, D. Elser, M. Lassen, U. L. Andersen, C. Marquardt and G. Leuchs, Naturally phase-matched second-harmonic generation in a whisperinggallery-mode resonator. Phys. Rev. Lett. 104, 153901 (2010) 
[40] J. A. Stratton, Electromagnetic theory (John Wiley \& Sons, Inc., New Jersey, 2007) Ch. 9.

[41] J. Van Bladel, Electromagnetic fields (McGraw-Hill Book Company, New York, 1964) Ch. 13. 


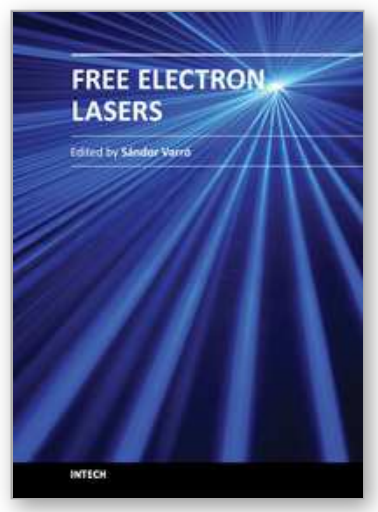

\author{
Free Electron Lasers \\ Edited by Dr. Sandor Varro
}

ISBN 978-953-51-0279-3

Hard cover, 250 pages

Publisher InTech

Published online 14, March, 2012

Published in print edition March, 2012

Free Electron Lasers consists of 10 chapters, which refer to fundamentals and design of various free electron laser systems, from the infrared to the xuv wavelength regimes. In addition to making a comparison with conventional lasers, a couple of special topics concerning near-field and cavity electrodynamics, compact and table-top arrangements and strong radiation induced exotic states of matter are analyzed as well. The control and diagnostics of such devices and radiation safety issues are also discussed. Free Electron Lasers provides a selection of research results on these special sources of radiation, concerning basic principles, applications and some interesting new ideas of current interest.

\title{
How to reference
}

In order to correctly reference this scholarly work, feel free to copy and paste the following:

Sándor Varró (2012). Interference Phenomena and Whispering-Gallery Modes of Synchrotron Radiation in a Cylindrical Wave-Guide, Free Electron Lasers, Dr. Sandor Varro (Ed.), ISBN: 978-953-51-0279-3, InTech, Available from: http://www.intechopen.com/books/free-electron-lasers/interference-phenomena-andwhispering-gallery-modes-of-synchrotron-radiation-in-the-presence-of-a-pe

\section{INTECH}

open science | open minds

\section{InTech Europe}

University Campus STeP Ri

Slavka Krautzeka 83/A

51000 Rijeka, Croatia

Phone: +385 (51) 770447

Fax: +385 (51) 686166

www.intechopen.com

\section{InTech China}

Unit 405, Office Block, Hotel Equatorial Shanghai

No.65, Yan An Road (West), Shanghai, 200040, China

中国上海市延安西路65号上海国际贵都大饭店办公楼405单元

Phone: +86-21-62489820

Fax: +86-21-62489821 
(C) 2012 The Author(s). Licensee IntechOpen. This is an open access article distributed under the terms of the Creative Commons Attribution 3.0 License, which permits unrestricted use, distribution, and reproduction in any medium, provided the original work is properly cited. 